\title{
Tell us where the data is
}

\section{A statement on the availability of data is now needed on all research published in Nature Climate Change.}

Understanding and addressing the challenges of climate change requires information from many scales. Research publications tell some of the story but the ability of others to find and use data may advance progress and allow greater insight to be gained.

Different research fields have different practices and approaches to sharing data. Overall there is a movement towards making data more available and useful, with specific requirements from some funders, publishers and institutions. For example, the Coalition on Publishing Data in the Earth and Space Sciences outlines a statement of commitment (http://www.copdess.org/). However, this is not yet the norm in many fields.

At Nature Climate Change and the Nature journals, our policy has always been that data must be made available by the authors on request. The full policy of the Nature journals can be found online ${ }^{1}$. Our policy now requires a statement to be included in the Methods section of accepted papers on the availability of the minimum data needed to understand, replicate and build on the publication. The inclusion of a statement regarding where this data can be found and accessed makes the process more transparent.

This policy is about stating the availability of data, but we are also encouraging authors to deposit their data in an appropriate repository. Our sister journal, Scientific Data - a data-descriptor journal - has a list of recommended repositories ${ }^{2}$. For authors whose data are in a repository and have been assigned a DOI, they are encouraged to cite the data set in the reference list. This in turn allows those who generated the data to get credit for their work.

What do these statements look like? They will vary depending on whether the data has been placed in a repository, is sourced from previous publications, or is held by the authors. Example statements can be found online ${ }^{3}$ along with guidance on how to write a statement.

This policy comes off the back of a trial at five Nature Research journals started in March this year. The journals span a wide range of disciplines. This has highlighted the different approaches to data sharing, and the variety in availability and use of public repositories across subjects. Since September, the policy has been gradually implemented across the whole portfolio of Nature journals. This is in line with a wider initiative at Springer Nature, with the introduction of a standardized set of data research policies ${ }^{4,5}$. The aim is to make our research data policy clearer to authors and readers, whilst making it straightforward for journals to adopt the policy that best suits their community.

We will be monitoring and working closely with our authors to ensure this new policy implementation runs smoothly. Our aim is to understand the needs of the diverse communities we serve and ensure we publish clear statements which benefit our readers, enhance our publications and help address the challenges ahead.

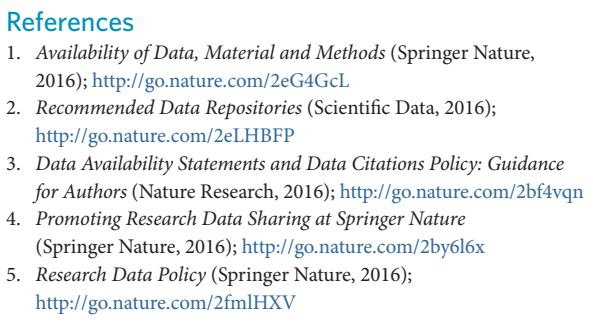

\section{Building a greener future}

\section{The built environment will play a key role in determining future emissions, so it is essential that low-carbon infrastructure and design are implemented.}

Globally, urbanization is rapidly increasing and as it does there will be a need for new infrastructure to accommodate the accompanying population growth. Cities and the urban environment are important drivers of greenhouse gas emissions as city design and infrastructure contribute to emissions. Reducing the carbon intensity of urban dwellers will play a large role in mitigation.

Cities already contribute to climate action. One example is the C40, a network of megacities collaborating to address climate change (http://www.c40.org/). Such leadership is welcome, and learning from others could greatly benefit rapidly expanding cities. However, there is no one-size-fits-all solution, with individual characteristics of cities affecting emissions reduction adaptations. An analysis of 22 global cities identified different approaches to fit different city types (C. A. Kennedy, N. Ibrahim \& D. Hoornweg,
Nat. Clim. Change 4, 343-346; 2014), suggesting cities must find solutions to fit their residents, climate and existing infrastructure.

In this issue, Felix Creutzig and co-authors (page 1054) discuss how existing and future urban infrastructure can play a role in urban climate solutions. They estimate buildings account for around 70\% of urban infrastructure emissions, with the remaining $30 \%$ from urban transport. Savings from changes to existing infrastructure can be achieved, but these are limited. The biggest emissions savings will be associated with new-built infrastructure. Construction of infrastructure results in emissions but it is the locked-in emissions, related to the use of infrastructure due to design and efficiency, that will have the greatest long-term reduction in emissions. Planning for low-carbon cities could reduce emissions from transport and buildings by $27-57 \%$.
A barrier to such adaptation is finance infrastructure and development requires investment and currently there are barriers to low-carbon investment. A Perspective from Ilmi Granoff and colleagues (page 1065) explores these barriers and how they might be overcome. The authors conclude that green growth requires an overall increase in infrastructure investments, whilst transitioning existing infrastructure investment towards low-carbon investment.

The urban environment changes slowly, but in some regions rapid urbanization is necessitating construction at an unprecedented rate. Globally $54 \%$ of the population now lives in cities (Urbanization and Development: Emerging Futures; UN-Habitat, 2016), contributing $70 \%$ of global greenhouse gas emissions, meaning urban design and planning for a low-carbon economy needs to start now. 